\title{
Evaluation of real-time QRS detection algorithms in variable contexts
}

\section{F. Portet}

IRISA - Institut de Recherche en Informatique et Systèmes Aléatoires And with

LTSI - Laboratoire Traitement du Signal et de l'Image Unité INSERM 642 Université de Rennes 1

Campus Beaulieu

35042 Rennes

France

France

Email: francois.portet@irisa.fr

\section{A.I. Hernández}

LTSI - Laboratoire Traitement du Signal et de l'Image Unité INSERM 642 Université de Rennes 1

Campus Beaulieu

35042 Rennes

France

France

Email: alfredo.hernandez@univ-rennes1.fr

G. Carrault (corresponding author)

LTSI - Laboratoire Traitement du Signal et de l'Image Unité INSERM 642 Université de Rennes 1

Campus Beaulieu

35042 Rennes

France

Phone: 0033 (0)2 23236594

Fax: 0033 (0)2 23236917

Email: guy.carrault@univ-rennes1.fr 
MS no 04/102

\section{Abstract}

A method is presented to evaluate the detection performance of real-time QRS detection algorithms to propose a strategy for the adaptive selection of QRS detectors, under variable signal contexts. Signal contexts are defined as different combinations of QRS morphologies and clinical noise. Four QRS detectors are compared under these contexts by means of a multivariate analysis. This evaluation strategy is general and can be easily extended to a larger number of detectors.

A set of morphology contexts, corresponding to 8 QRS morphologies (Normal, PVC, premature atrial beat, paced beat, $\mathrm{LBBB}$, fusion, RBBB, junctional premature beat), has been extracted from 17 standard ECG records. For each morphology context, the set of extracted beats, ranging from 30 to 23000 , are resampled to generate 50 realizations of 20 concatenated beats. These realizations are then used as input to the QRS detectors, without noise, and with 3 different types of additive clinical noise (electrode motion artefact, muscle artefact, baseline wander) at 3 signal-to-noise ratios $(5 \mathrm{~dB},-5 \mathrm{~dB},-15 \mathrm{~dB})$. Performance is assessed by the number of errors, which reflects both false alarms and missed beats.

The results show that the evaluated detectors are indeed complementary. For example, the Pan and Tompkins's detector is the best in most contexts but the Okada's detector generates less errors in presence of electrode motion artefact. These results will be particularly useful to the development of a real-time system that will be able to choose the best QRS detector according to the current context.

Keywords: ECG analysis, QRS detection, algorithms evaluation, best algorithm selection, real time signal monitoring 
MS no 04/102

\section{Introduction}

Electrocardiogram (ECG) analysis is an important tool in the management of cardiac diseases. One of the most relevant tasks in automatic ECG analysis is the detection and characterization of every wave, particularly of the QRS complex, after which a more complete analysis can be obtained (PASSARIELLO et al., 1993; CARRAULT et al., 2003). Therefore, choosing the QRS detection algorithm is an essential step in the development of a real-time ECG analysis system. Experience, over several years, shows that the proposed strategies for ECG analysis and particularly for QRS complex detection based on signal processing techniques, have reached an asymptotic detection performance. This is mainly due to the multiplicity of situations met in clinical environments. An alternative would be to use the best detection algorithm, according to the current context. A first step to reach this objective is to develop an evaluation methodology to compare different QRS detectors under a combination of noise and QRS morphologies.

Some quantitative performance comparisons of QRS detectors are presented in the literature (POLI et al., 1995; KADAMBE et al., 1999; BENITEZ et al., 2001). These evaluations are usually performed on a set of records extracted from standard ECG databases (such as the MIT-BIH, CSE, AHA, ...) and are based on detection scores, generally expressed as sensitivity-specificity pairs. An average score, calculated over a set of different records, is assumed to reflect the overall performance of the detectors. A limitation of this method is that an ECG is composed of multiple noise levels and types, and a variety of beat morphologies. As reported by KOHLER et al. (2002), an average score hides the problems that are still present in case of noisy or pathological signals because it does not explain what are the specific features of the ECG that affect the detection. Moreover, a reliable comparison implies that QRS detectors performance evaluation must be done from the same test signal database, which is not the case in the literature.

A good example of a study comparing QRS detectors is FRIESEN et al. (1990). They compared nine simple QRS detection algorithms with respect to a gold standard ECG waveform. This waveform was corrupted with five types of artificial noise, modelling typical clinical noise. The authors concluded on the best of the nine QRS detectors, by comparing their average performance and clearly exhibited that each detector performance is related to the noise context. However, this study does not taken into account neither the filtering stage, nor the effect of QRS morphologies. Other studies have taken into account the influence of clinical noise, such as in SUN et al. (2002) with simulated noise or MoODY et al. (1984) with experimental noise. Nevertheless, the influence of QRS morphologies on the detector performance was not evaluated. NYGÅRDS and SÖRNMO (1981) studied the distance between manual and automatic QRS delineation methods, adding gaussian noise at different signal-to- 


\section{MS no 04/102}

noise ratios, but this work was limited to only two QRS morphologies and two different methods (manual and automatic). This brief state of the art shows that current comparisons, reported in the literature, do not evaluate the QRS detectors sensitivity to the global signal context (beat morphology, noise, etc.).

This work presents a methodology to compare a set of QRS detectors under different noise conditions and QRS morphologies, in order to determine the best detector for a given context. Such an evaluation would be useful for the inference of general algorithm selection rules for on line monitoring. A signal context is defined here as a particular combination of noise and QRS morphologies. A change of the noise energy or a change of the QRS morphology within an ECG record implies a change of context. To create different context signals, typical clinical noise and QRS morphologies were extracted from actual ECG records. Since the filtering stage is taken into account in every real application of QRS detection, the ECG waveform was filtered in order to reduce noise. The tested detectors were chosen considering both complexity and efficiency but prioritising the low complexity against the efficiency, since the paper is devoted to real-time cardiac monitoring.

The next section introduces the databases, the filtering stage, and the evaluated QRS detection algorithms. Then, the method to generate the context database, considering both noise and QRS morphologies, is described. In the same section, the scoring criterion, which is the number of errors generated under each context, is detailed. Finally, the results of the principal component analysis on the score data are presented.

\section{Material}

\subsection{The databases}

ECG signals were excerpted from the MIT-BIH Arrhythmia Database (MBAD) (MARK and MoODY, 1988). The clinical additive noise was extracted from the MIT-BIH Noise Stress Test Database (MooDY et al., 1984). Noise was recorded on physically active volunteers using standard ECG recorders, leads, and electrodes in positions in which the ECG of the subjects was not visible. Three noise records were generated (see Fig. 1, b, d, and f), by selecting intervals that contained predominantly baseline wander (bw), muscle (EMG) artefact (ma), and electrode motion artefact (em).

\subsection{Filtering stage}

It is based on the work of SENHADJI et al. (1992). Succinctly, using a multiresolution analysis, the signal is reconstructed from only the details corresponding to the QRS complex spectrum band (1.9, $45 \mathrm{~Hz})$. This step is very important because the filter distorts the ECG signal (JENKINS and CASWELL, 1996) and consequently, the performance of the QRS detectors depends also on the quality of the pre-processing filter (Fig. 1, e). 
MS no 04/102

--Figure 1--

\subsection{Evaluated QRS detection Algorithms}

Many QRS detection schemes are described in the literature (OKADA, 1979; FRADEN and NEUMAN, 1980; PAN and TOMPKINS, 1985; GRITZALI, 1988; POLI et al., 1995; SillPO and MARCHESI, 1998; KADAMBE et al., 1999; WIEBEn et al., 1999; DASKALOV and CHRISTOV 1999) and are still being proposed (BENITEZ et al., 2001; BURKE and NASOR, 2004; DOTSINSKY and STOYANOV, 2004). Since it would be impracticable to compare all of them, only four were selected according to these criteria: ability to work in real-time, ease of implementation, robustness to noise, and knowledge of their performance in noiseless situations. The four chosen detectors were those proposed by PAN and TOMPKINS (1985) (P\&T), GRITZALI (1988), FRADEN and NEUMAN (1980), and OKADA (1979). FREISEN et al. (1990) presented those of FRADEN and NEUMAN (1980), and OKADA (1979) as AF2 and DF2 in their study; they demonstrated that these detectors are sensitive to different types of noise and are, in this sense, complementary. This selection enabled us to compare our results to those of FRIESEN et al. (1990).

\section{Method}

This section describes the generation of the context signals and the scoring criteria.

\subsection{Context generation}

To build the test signals database, different types of QRS morphologies were combined with additive clinical noise. The original QRS waveforms were extracted from 17 records of the annotated MBAD. These records were chosen because of their recording quality and the presence of different QRS morphologies. To simulate the real time buffer, segments of 20 QRS, usually around 23 seconds, were built. This corresponds to the typical length of 8192 samples (FRIESEN et al., 1990) for a sampling frequency of $360 \mathrm{~Hz}$. Only the data recorded by the Modified Lead II (MLII) were used.

Firstly, we construct the context called the morphology contexts. Eight QRS morphology signals, containing complexes of one type only, ranging from 30 to 23000 QRS segments, were extracted from 17 MBAD records (Tab. 1). To preserve the RR interval between two adjacent QRS, the samples of the $n^{\text {th }} \mathrm{QRS}$ segment were extracted from the original record interval: 
MS no 04/102

$$
\left[\frac{R(n)+R(n-1)}{2}, \frac{R(n)+R(n+1)}{2}-1\right]
$$

where $R(n)$ is the date of the $n^{\text {th }} \mathrm{R}$ wave (peak or major local extremum) in the MBAD annotations. A pseudobootstrapping method adapted from ZOUBIR and BOASHASH (1998) is performed to construct a larger data set from this limited number of individuals and to derive 400 morphology context signals of 20 concatenated QRS segments.

Secondly, the morphology contexts were corrupted by three types of real additive noise (bw, ma, em) at three Signal-to-Noise Ratios (SNR): 5, -5, -15 dB. In this application, the SNR is computed by the following formula:

$$
S N R=10 \cdot \log _{10}\left(\frac{P(S)}{P(\alpha \cdot N)}\right) \Rightarrow \alpha=\sqrt{\frac{P(S)}{P(N)} \times 10^{\frac{-S N R}{10}}}
$$

where the variable $\alpha$ controls the power of the noise and $P(X)$ is the power of $X$. To remove as much original noise as possible, the original ECG was filtered, as described in 2.2 , before the addition of the noise. Uncorrupted morphology contexts were also tested. A total of 4000 realizations for 80 contexts were obtained. A typical context is shown in Figure 1 (c).

\section{-- Table 1 --}

\subsection{Scoring criterion}

The evaluated QRS detection algorithms are based on a non-linear transformation of the ECG signal, followed by thresholding. Only the transformation stage of each algorithm was applied to each buffer of 20 QRS. To better compare the detection results, a set of threshold values was chosen for each algorithm under each context, rather than using the original threshold definitions. For further details about these algorithms, the reader is invited to consult the corresponding references.

An event is detected if a segment of the signal is above the threshold $\tau$ and the occurrence of this event is the date of the sample presenting the maximum amplitude in this segment. If an event belongs to a window of 128 ms around the QRS annotation, then it is labelled as a True Positive (TP), otherwise it is labelled as a False Positive (FP). If two or more events belong to a same window, only one is labelled TP, the others are ignored. False Negatives (FN) are the QRS annotations not associated to any event (i.e. the event is missed). The probability of detection $(\mathrm{Pd})$ and the probability of false alarm (Pfa) are computed as:

$$
P d(\tau)=\frac{N_{T P}(\tau)}{N_{T P}(\tau)+N_{F N}(\tau)}, \quad P f a(\tau)=\frac{N_{F P}(\tau)}{N_{T}}
$$




\section{MS no 04/102}

where $N$. is the number of $T P, F N, F P$ for the given threshold $\tau$, and $N_{T}$ is the maximum number of false alarms for the whole set of thresholds under the hypothesis of QRS absence. The scoring criterion, to evaluate the detectors, is the number of errors $(\mathrm{Ne})$ defined as:

$$
N e=N_{F P}\left(\tau^{*}\right)+N_{F N}\left(\tau^{*}\right)
$$

where $\tau *$ is the optimal threshold value, estimated from Receiver Operating Characteristics curves (i.e. the Pd versus Pfa curves), and corresponds to the nearest point of the ideal detection point (i.e. $\mathrm{Pfa}=0 ; \mathrm{Pd}=1$ ) (HERNÁNDEZ et al., 1999).

\subsection{Analysis of the results}

Ne values were calculated for all the combinations of R signal realizations, D detectors, $\mathrm{M}$ morphologies and $\mathrm{N}$ types of noise totalling RxDxMxN Ne values. The number of Ne values grows considerably with the number of contexts and the interesting results could be difficult to analyse in such an amount of data. That is why a Principal Components Analysis (PCA) was performed to analyse the results. The aim of PCA is to represent a large set of multidimensional data in a smaller space, by maximizing the inertia for each orthogonal direction (KRZANOWSKI, 1998).

If $\mathbf{M}_{\mathbf{n}, \mathbf{p}}$ is a matrix of $n$ statistical individuals described by $p$ quantitative variables, $\mathbf{X}_{\mathbf{n}, \mathbf{p}}$ is a matrix of $\mathbf{M}_{\mathbf{n}, \mathbf{p}}$ where the variables are reduced-centred. In this particular case, the eigenvectors $\mathbf{a}_{\mathbf{p}, \mathbf{1}}^{\mathbf{k}}$ of $\mathbf{X}^{\mathrm{T}} \mathbf{X}, \forall \mathrm{k} \in\{1,2, \ldots, \mathrm{p}\}$, are the principal factors and $\zeta_{\mathbf{n}, 1}^{\mathbf{k}}=\mathbf{X}_{\mathbf{n}, \mathbf{p}} \mathbf{a}_{\mathbf{p}, \mathbf{1}}^{\mathbf{k}}, \forall \mathrm{k} \leq \mathrm{p}$ is the $\mathrm{k}^{\text {th }}$ principal component that corresponds to the individuals of $\mathbf{X}$ projected on the $\mathrm{k}^{\text {th }}$ orthogonal axis (i.e. the coordinates). The explained variance $\varphi^{\mathrm{k}}$ of the $\mathrm{k}^{\text {th }}$ axis is equal to $\lambda^{\mathrm{k}} / \mathrm{p}, \varphi^{\mathrm{k}} \in[0,1],\left(\Sigma \lambda^{\mathrm{k}} / \mathrm{p}=1\right)$ where $\lambda^{\mathrm{k}}$ is the $\mathrm{k}^{\mathrm{th}}$ eigenvalue of $\mathbf{X}^{\mathrm{T}} \mathbf{X}$. The higher the value of $\varphi^{\mathrm{k}}$, the better the $\zeta^{\mathbf{k}}$ resumes the $\mathrm{p}$ variables of $\mathbf{X}$.

In this application, the matrix to analyze is constituted of $\mathrm{Ne}$ values of the 4 detectors, for all combinations of QRS morphologies and noise levels and types. Values of $\zeta_{\mathbf{n}, \mathbf{1}}^{\mathbf{k}}$ are thus a linear combination of the reducedcentred version of this matrix by the corresponding eigenvector $\mathbf{a}_{\mathbf{p}, \mathbf{1}}^{\mathbf{k}}$, representing a synthesis of all the variables. In this sense, each axis reflects a particular combination of the original $\mathrm{Ne}$ data. The most predominant $\mathrm{Ne}$ variables for a given axis $\mathrm{k}$, can be identified by calculating the correlation between the $\mathrm{k}^{\text {th }}$ principal factor $\mathbf{a}^{\mathbf{k}}$ and the $\mathrm{j}^{\text {th }}$ variable $\mathbf{x}^{\mathbf{j}}$ : $\operatorname{Corr}\left(\mathbf{a}^{\mathbf{k}}, \mathbf{x}^{\mathbf{j}}\right)=\mathbf{a}_{\mathbf{j}}^{\mathbf{k}} \sqrt{ } \lambda^{\kappa}$ and by selecting the highest correlation values. 
Init. Experiment: For a given morphology context, suppose a set $\mathrm{Q}$ of $\mathrm{B}_{\mathrm{T}}$ beats of $\mathrm{K}$ samples.

Step 1 Resampling: Draw a random selection of $B$ beats $(B=20)$, with replacement, from $Q$ to obtain the resampled population $\mathrm{Q}^{*}$.

Step 2 Linking: Concatenate the B beats of Q* using a sigmoid function on several samples so as to minimize the baseline shift between two adjacent beat segments as in WENDLING et al. (1997).

Step 3 Addition of noise: Corrupt the concatenated beats by real additive noise at a specific Signal-to-Noise Ratio to obtain S. The added noise segment is chosen randomly from a specific noise record of the MIT noise stress database. For the generation of uncorrupted morphology contexts, the step 3 is ignored.

Step 4 Filtering: Filter S using the filter described in section 2.2 (filtering stage) to obtain the signal S'.

Step 5 Calculation of the score: Use S' as input of each one of the detectors. The score is the number of error (Ne) computed using (1).

Step 6 Repetition: Repeat steps 1 to 5 to obtain $\mathrm{R}$ realizations $(\mathrm{R}=50)$ of the same context.

Step 7 Repetition: Repeat the algorithm for each given context to collect all the scores.

Step 8 Presentation of the results: Project all the scores on principal axes (PCA) to visually analyse their dispersion.

\section{Results}

Ne values were calculated for all the combinations of the 50 signal realizations, 4 detectors, 8 morphologies and 10 types of noise, totalling $16000 \mathrm{Ne}$ values. These Ne values were organized into two different matrices:

- M1: This matrix is composed of Ne values for the 4 QRS detectors, applied to the 50 signal realizations, (200 rows) and 80 columns, corresponding to the combinations of the 8 morphologies and 10 types of noise. This forms a matrix of 200 individuals described by 80 variables.

- M2: Rows are the 50 realizations of each context $(50 \times 8 \times 10=4000)$ and columns are the four detectors. This forms a matrix of 4000 individuals described by 4 variables.

PCA has been applied to these matrices in order to study: 1) the dependence of detector performance with respect to the contexts (PCA of M1) and 2) the contexts according to the detectors performance (PCA of M2).

For each PCA, four particular context type groups have been analysed: the QRS morphology context (i.e. contexts without noise), the baseline wander noise context (bw at all SNR), the muscle artefacts context (ma at all SNR), and the electrode motion artefact context (em at all SNR). In each case, the two principal components (i.e. the projected individuals) were plotted, according to their explained variation. For each group of context types, different symbols were used and two properties were looked after: the barycentre, which gives information about the quality of the detection (plotted in bold black) and the dispersion, which gives information about the 


\section{MS no 04/102}

robustness of the detection. To interpret the meaning of the PCA axes, examples of subsets of correlation values for the two main axes along the initial variables are shown in Tab. 2 and Tab. 3 .

\subsection{Detectors performance according to the contexts (M1)}

Five PCA were performed on M1, one for the global data matrix, and one for each context type. Concerning PCA on global contexts (Fig. 2), the first axis is mostly correlated to the Ne values of ma noise at low SNR levels, while the second axis is correlated to the Ne values of ma noise contexts versus em noise contexts (see correlations Tab. 2). The first axis $\left(\varphi^{1}=25.6 \%\right)$ shows that $\mathrm{P} \& \mathrm{~T}$ and Gritzali are superior to $\mathrm{AF} 2$ and $\mathrm{DF} 2$. The second axis $\left(\varphi^{2}=11.6 \%\right)$ shows that $\mathrm{AF} 2$ is particularly sensitive to em noise, DF2 is particularly sensitive to ma noise, and P\&T and Gritzali are less sensitive to noise than DF2 and AF2 (coordinates near zero). In order to obtain a comparison of these detectors on specific contexts, four additional PCA have been performed:

- PCA on QRS morphology contexts $\left(\varphi^{1}=32.8 \%\right.$ and $\left.\varphi^{2}=13.6 \%\right)$ : It emphasized the dispersion of AF2 and DF2, whereas the Gritzali and P\&T present similar low Ne values.

- $\quad$ PCA on bw noise contexts $\left(\varphi^{1}=26.2 \%\right.$ and $\left.\varphi^{2}=9.76 \%\right)$ : It showed the particularly poor performance of AF2.

- PCA on ma noise contexts $\left(\varphi^{1}=43.8 \%\right.$ and $\left.\varphi^{2}=17 \%\right)$ : This analysis showed that detectors are well separated. The P\&T is the best according to its low Ne.

- PCA on em noise contexts: The first axis is related to the medium SNR level contexts while the second axis is correlated to low SNR values (Tab. 3). P\&T and Gritzali present low Ne on the first axis $\left(\varphi^{1}=21.8 \%\right)$ but are well separated on the second axis $\left(\varphi^{2}=15.2 \%\right)$ where DF2 shares particularly low Ne with P\&T (Fig. 3).

-- Table 2 --

-- Figure 2 --

-- Table 3 --

-- Figure 3 --

\subsection{Contexts influence on the detectors performance (M2)}

PCA on morphology contexts (Fig. 4) emphasizes the differences between the detection performance of premature Ventricular contraction beats $(\mathrm{V})$, Fusion of ventricular and normal beats (F), Left bundle branch block beats (L), and the rest of the QRS morphologies. For the first axis $\left(\varphi^{1}=60.8 \%\right)$, the correlation 
MS no 04/102

$\operatorname{Corr}\left(\mathbf{a}^{1}, \mathbf{X}\right)=[-0.53-0.84-0.84-0.86]^{\mathrm{T}}$, where the values are respectively associated to the variables of $\mathbf{X}$ [AF2 DF2 Gritzali P\&T], indicates that the first axis represents the global performance of all detectors. The barycentre coordinate on the first axis of the $\mathrm{V}$ beat is negative $(-3.43 \pm 0.99)$ and its high dispersion shows the lack of robustness of all the detectors for this QRS morphology. The barycentre and the dispersion of the F beat coordinate $(-0.24 \pm 0.67)$ point out the difficulty to detect this kind of beat. The $\mathrm{R}(0.89 \pm 0.02)$ and $\mathrm{J}(0.81 \pm 0.11)$ beats present the lowest dispersions. The second axis $\left(\varphi^{2}=20.4 \%\right)$ is mainly correlated to AF2 $\left(\operatorname{Corr}\left(\mathbf{a}^{2}, \mathbf{X}\right)=[-\right.$ $\left.\begin{array}{llll}0.84 & 0.01 & 0.21 & 0.23\end{array}\right]^{\mathrm{T}}$ ) and enhances the poor detection performance of this detector for the L beat.

\section{-- Figure 4 --}

Similar tests were performed for baseline wander noise, muscle artefact, and electrode motion artefact at different SNR levels $(5 \mathrm{~dB},-5 \mathrm{~dB},-15 \mathrm{~dB})$. These results show that bw does not affect significantly the detector sensitivity while, in presence of ma or em, the detector sensitivity becomes highly signal-to-noise dependent. More specifically, the P, V, and L beats are harder to detect with em and ma noise than the other beats whatever the SNR level.

\subsection{Overall analysis and discussion}

PCA results highlight the interesting data present in the raw results. A low average number of errors $(\mathrm{Ne}<0.19)$ is observed for the $\mathrm{R}$ and $\mathrm{J}$ beats for uncorrupted QRS waveforms. A medium score is estimated for the QRS complexes corresponding to the $\mathrm{N}, \mathrm{A}$, and $\mathrm{P}$ types $(0.2<\mathrm{Ne}<0.4)$ while $\mathrm{V}$ and $\mathrm{F}$ beats cause a high number of errors $(\mathrm{Ne}>1.4)$. The ma noise decreases all the performances and the differences induced by the morphologies are no longer perceivable at $-15 \mathrm{~dB}$. The em noise is quite disruptive and emphasizes the dispersion of QRS detection for different morphologies.

AF2 and DF2 show a lot of disparity in the results according to the QRS morphologies. AF2 is better than DF2 for $\mathrm{N}$ beat and DF2 is the best for $\mathrm{A}, \mathrm{P}, \mathrm{L}, \mathrm{R}$ and $\mathrm{J}$ beats. But both are very ineffective for $\mathrm{V}(\mathrm{Ne}>5)$ and $\mathrm{F}$ (Ne>2.45) QRS complexes. AF2 presents particularly poor performance (Ne>9) for L beats. As FRIESEN et al. (1990), we observed that DF2 is better than AF2 in presence of bw noise while AF2 is better than DF2 in presence of ma noise. Moreover, our study shows that DF2 is still better than AF2 in the presence of em noise. P\&T is the best detector (partly because of its band pass filter) whatever the morphology of the QRS complex, excepted for the $\mathrm{V}$ and the $\mathrm{F}$ morphologies, without noise and with bw noise, where Gritzali exhibits a better detection performance. However, both algorithms show a very poor performance for the $\mathrm{V}$ beats $(\mathrm{Ne}>3)$. In presence of em noise, P\&T could be substituted by DF2 for several QRS morphologies (N, A, V, Paced). 
MS no 04/102

A limitation of this study is related to the fact that the $\mathrm{P}, \mathrm{L}, \mathrm{R}$, and $\mathrm{J}$ beats have been extracted from less than three patients, whereas the others beats have been obtained from five patients at least (see Table 1). This could occasion a bias in the study of the generated morphology contexts. However, results emphasize that the performance of the evaluated detector depends on the signal context. These results could be used to state algorithm selection rules such as:

$$
\begin{aligned}
& \text { If < noise is bw or no noise > and <morphology is likely to be } \mathbf{V} \text { or } \mathbf{F}>\text { then use Gritzali } \\
& \text { If }<\text { noise is em at }-15 \mathrm{~dB}>\text { and }<\text { morphology is likely to be } \mathbf{N} \text { or } \mathbf{A} \text { or } \mathbf{V} \text { or Paced }>\text { then use DF2 }
\end{aligned}
$$

\section{Conclusion}

This paper has presented a new method for comparing QRS detectors, which takes into account the direct consequences on detection performance due to beat morphologies (and their corresponding differences in frequency contents) and clinical noise. The major goal has been to propose a robust methodology to determine which is the best QRS detector in a particular context. The results show that the evaluated QRS detectors are complementary. For example, the Pan and Tompkins's detector is the best in most contexts but the Okada's detector generates less errors in presence of electrode motion artefact.

The consequences of the transition between two different QRS morphologies were not studied but could be assesed to improve the method. Even if the study is limited to simple algorithms here, the method is general and other detectors such as those recently proposed (BENITEZ et al., 2001; BURKE and NASOR, 2004; DOTSINSKY and STOYANOV, 2004) and described by KOHLER et al. (2002), could be evaluated in the same manner. Moreover, the methodology is not restricted to the QRS complex and could be applied to other ECG signal waves (P waves detection algorithms) or to other biomedical signals such as EEG waves detection (spikes detection algorithms). This work shows that an improved QRS detection performance can be obtained by selecting the most appropriate algorithm for a given context (SHEKHAR et al. , 1994) based upon, for example, the selection rules proposed above. Obvioulsy, the implementation of this kind of approach depends on the correct estimation of the current signal context (noise and expected QRS morphologies). This is part of our current work that will lead us to the development of a strategy of QRS detector driving in real time monitoring. 
MS no 04/102

\section{Acknowledgments}

The authors wish to thank Pr. M.-O. Cordier, Dr. R. Quiniou and Dr. E. Roux, for their thoughtful comments. 
MS no 04/102

\section{References}

Benitez, D., Gaydecki, P., Zaidi, A., And FitzPatrick, A. (2001): 'The use of the Hilbert transform in ECG signal analysis', Comput. Biol. Med., 31, pp. 399-406

BurKe, M., AND NASOR, M. (2004): 'Wavelet based analysis and characterization of the ECG signal', J. Med. Eng. Technol., 28(2), pp. $47-55$

Carrault, G., Cordier, M.-O., Quiniou, R., AND Wang, F. (2003): ‘Temporal abstraction and inductive logic programming for arrhythmia recognition from electrocardiograms', Artif. Intell. Med., 28, pp. 231-263

DASKalov, I. K., AND Christov, I.I. (1999): 'Electrocardiogram signal preprocessing for automatic detection of QRS boundaries', Med. Eng. Phys., 21, pp. 37-44

Dotsinsky, I.A., AND Stoyanov, T.V. (2004): 'Ventricular beat detection in single channel electrocardiograms', Biomed. Eng. Online, 3(1)

Fraden, J., And Neuman, M. (1980): 'QRS wave detection', Med. Biol. Eng. Comput., 18, pp. 125-132

Friesen, G., JannetT, T., Jadallah, M., Yates, S., Quint, S., AND NAGLe, H. (1990): ‘A comparison of the noise sensitivity of nine QRS detection algorithms', IEEE Trans. Biomed. Eng., 37, pp. 85-98

GRITZALI, F. (1988): 'Towards a generalized scheme for QRS detection in ECG waveforms', Signal Process., 15, pp. 183192

Hernández, A.I., Carrault, G., Mora, F., Thoraval, L., Passariello, G., and Schleich, J.M. (1999): 'Multisensor fusion for atrial and ventricular activity detection in coronary care monitoring', IEEE Trans. Biomed. Eng., 46(10), pp. $1186-1190$

Jenkins, J.M., AND CASwell, S.A. (1996): 'Detection algorithms in implantable cardioverter defibrillators', Proc. of the IEEE, 84(3), pp. 428-445

Kadambe, S., Murray, R., AND BoudreauX-Bartels, F. (1999): 'Wavelet transform-based QRS complex detector', IEEE Trans. Biomed. Eng., 46(7), pp. 838-848

Kohler, B.-U., Hennig, C., AND Orglmeister, R. (2002): ‘The principles of software QRS detection', IEEE Eng. Med. Biol. Mag., 21(1), pp. 42-57

KrZANOwsKi, W. (1998): 'Principles of multivariate analysis', AtKinson, A.C., CoPAS, J.B., PIERCE, D.A., SCHERvish, M.J., AND TitTERINGTOn, D.M. (Eds) ‘Oxford Statistical Science Series’, Oxford University Press, Oxford, U.S., pp. 53-??

MARK, R., AND MoOdy, G. (1988): ‘MIT-BIH arrhythmia data base directory', Massachusetts Institute of Technology

Moody, G., Muldrow, W., And MARK, R. (1984): ‘A noise stress test for arrhythmia detectors', Comput. Cardiol., 11, pp. $381-384$

NYGÅRDS, M.-E., AND SÖRNMO, L., (1981): 'A QRS delineation algorithm with low sensitivity to noise and morphology changes', Proc. IEEE Comput. Cardiol., Florence, Italy, pp. 347-350

OKADA, M. (1979): ‘A digital filter for the QRS complex detection', IEEE Trans. Biomed. Eng., BME-26, pp. 700-703 


\section{MS no 04/102}

PAN, J., AND TOMPKINS, W. J. (1985): ‘A real-time QRS detection algorithm', IEEE Trans. Biomed. Eng., BME-32(3), pp. $230-236$

Poli, R., CAgnoni, S., ANd VAlli, G. (1995): ‘Genetic design of optimum linear and nonlinear QRS detectors', IEEE Trans. Biomed. Eng., 42(11), pp. 1137-1141.

Passariello G., Mora F., Carrault G., and Le Pichon J.P. (1993): 'Intelligent patient monitoring and management systems: a review', IEEE Eng. Med. Biol .Mag., 12(4), pp. 23-33.

Senhadji, L., Carrault, G., Bellanger, J.J., and Passariello, G. (1992): 'Some new applications of the wavelet transforms', 14th An. Int. Conf. IEEE-EMBS, Paris, France, 14(6), pp. 2592-2593

SheKhar, C., Moisan, S., And Thonnat, M. (1994): 'Towards an intelligent problem-solving environment for signal processing', Math. Comput. Simul., 36, pp. 347-359

SiLIPO, R., AND MARCHESI, C. (1998): ‘Artificial neural networks for automatic ECG analysis', IEEE Trans. Signal Process., 46(5), pp. 1417-1425

Sun, Y., Chan, K., ANd Krishnan, S. (2002): 'ECG signal conditioning by morphological filtering', Comput. Biol. Med., 32, pp. $465-479$

Wendling, F., Carrault, G., AND Badier, J.M. (1997): 'A comparative study of depth EEG seizure signals: Proposition for a method based on a physiologically relevant parameter', Annal of Biom. Eng., 25, pp. 1026-1039

Wieben, O., AfOnSO, V.X., AND TOMPKINS, W.J. (1999): 'Classification of premature ventricular complexes using filter bank features, induction of decision trees and a fuzzy rule-based system', Med. Biol. Eng. Comput., 37(5), pp. 560-565

ZoubIR, A.M., AND BOASHASH, B. (1998): 'The bootstrap and its application in signal processing', IEEE Signal Process.

Mag., 15(1), pp. 56-76 
MS no $04 / 102$

Table 1 The morphology contexts database

\begin{tabular}{|c|c|c|c|}
\hline QRS waveform & Symbol & $\begin{array}{l}\text { Number } \\
\text { of QRS }\end{array}$ & MIT-BIH records \\
\hline Normal beat & $\mathrm{N}$ & 23703 & $\begin{array}{c}100,101,103,106,113,115,119, \\
122,123,201,205,231,233\end{array}$ \\
\hline $\begin{array}{c}\text { Atrial } \\
\text { premature beat }\end{array}$ & A & 81 & $\begin{array}{c}100,101,103,124,201,205,231, \\
233\end{array}$ \\
\hline $\begin{array}{l}\text { Premature } \\
\text { ventricular } \\
\text { contraction }\end{array}$ & V & 2216 & $\begin{array}{c}100,101,106,107,109,111,119 \\
123,124,201,205,231,233\end{array}$ \\
\hline Paced beat & $\mathrm{P}$ & 2078 & 107 \\
\hline $\begin{array}{l}\text { Left Bundle } \\
\text { Branch Beat }\end{array}$ & $\mathrm{L}$ & 4615 & 109,111 \\
\hline $\begin{array}{l}\text { Fusion of } \\
\text { ventricular and } \\
\text { normal beat }\end{array}$ & $\mathrm{F}$ & 31 & $109,124,201,205,233$ \\
\hline $\begin{array}{l}\text { Right Bundle } \\
\text { Branch Beat }\end{array}$ & $\mathrm{R}$ & 2785 & 124,231 \\
\hline $\begin{array}{c}\text { Jonctional } \\
\text { premature beat }\end{array}$ & $\mathrm{J}$ & 30 & 124,201 \\
\hline
\end{tabular}


MS no 04/102

Table 2 Correlation between some variables and the $1^{\text {st }}$ and $2^{\text {nd }}$ axes $\left(a^{l}\right.$ and $\left.a^{2}\right)$ for the global context (first

$P C A)$

\begin{tabular}{|c|c|c|c|c|c|c|c|c|c|c|c|c|c|}
\hline \multirow{4}{*}{ Axis 1} & & Morphology* & $\mathrm{L}$ & V & $\mathrm{P}$ & $\mathrm{N}$ & $\mathrm{F}$ & $\mathrm{P}$ & $\mathrm{P}$ & A & $\mathrm{P}$ & $\mathrm{J}$ & .. \\
\hline & $\mathrm{x}^{\mathrm{j}}$ & Type of noise & $\mathrm{ma}$ & $\mathrm{ma}$ & $\mathrm{ma}$ & $\mathrm{ma}$ & $\mathrm{ma}$ & bw & em & $\mathrm{ma}$ & bw & $\mathrm{ma}$ & $\ldots$ \\
\hline & & SNR (dB) & -15 & -5 & -15 & -15 & -15 & -5 & 5 & -15 & 5 & -15 & ... \\
\hline & Cor & $r\left(a^{1}, x^{j}\right)$ & 0,86 & 0,86 & 0,86 & 0,81 & 0,81 & 0,77 & 0,77 & 0,77 & 0,77 & 0,77 & $0,77>\ldots$ \\
\hline \multirow{4}{*}{ Axis 2} & & Morphology & A & $P$ & $\mathrm{R}$ & $\mathrm{L}$ & $\mathrm{N}$ & $\mathrm{P}$ & $\mathrm{J}$ & $\ldots$ & $\mathrm{N}$ & V & J \\
\hline & $x^{j}$ & Type of noise & $\mathrm{ma}$ & $\mathrm{ma}$ & $\mathrm{ma}$ & $\mathrm{ma}$ & $\mathrm{ma}$ & $\mathrm{ma}$ & $\mathrm{ma}$ & $\ldots$ & em & em & em \\
\hline & & SNR (dB) & 5 & 5 & -5 & -5 & -5 & -5 & 5 & $\ldots$ & -15 & -5 & -5 \\
\hline & Cor & $r\left(a^{2}, x^{j}\right)$ & 0,79 & 0,73 & 0,73 & 0,67 & 0,67 & 0,64 & 0,61 & $\ldots$ & $-0,55$ & $-0,61$ & $-0,61$ \\
\hline
\end{tabular}

* See Tab. 1 for QRS type definition. 
MS no 04/102

Table 3 Correlation between some variables and the $1^{\text {st }}$ and $2^{\text {nd }}$ axes $\left(a^{1}\right.$ and $\left.a^{2}\right)$ for the electrode motion artefact context

\begin{tabular}{|c|c|c|c|c|c|c|c|c|c|c|c|c|c|}
\hline \multirow{3}{*}{ Axis 1} & \multirow{2}{*}{$x^{j}$} & Morphology & $\mathrm{P}$ & $\mathrm{N}$ & $\mathrm{V}$ & $\mathrm{J}$ & $\mathrm{V}$ & $\mathrm{R}$ & $\mathrm{F}$ & $\mathrm{L}$ & $\mathrm{P}$ & $\mathrm{J}$ & $\ldots$ \\
\hline & & $\mathrm{SNR}(\mathrm{dB})$ & 5 & -5 & -5 & 5 & 5 & 5 & 5 & 5 & -5 & -5 & $\ldots$ \\
\hline & \multicolumn{2}{|c|}{$\operatorname{Corr}\left(a^{1}, x^{j}\right)$} & 0,89 & 0,73 & 0,71 & 0,69 & 0,64 & 0,55 & 0,53 & 0,48 & 0,46 & 0,43 & $0,41>$ \\
\hline \multirow{3}{*}{ Axis 2} & & Morphology & $\mathrm{J}$ & $\mathrm{N}$ & $\mathrm{L}$ & A & $\mathrm{J}$ & F & $\mathrm{L}$ & A & $\mathrm{R}$ & $\mathrm{P}$ & $\ldots$ \\
\hline & & $\mathrm{SNR}(\mathrm{dB})$ & -15 & -15 & -5 & -5 & -5 & -15 & -15 & -15 & -15 & -15 & $\ldots$ \\
\hline & & $\operatorname{rr}\left(a^{2}, x^{j}\right)$ & 0,67 & 0,62 & 0,60 & 0,60 & 0,56 & 0,53 & 0,51 & 0,41 & 0,40 & 0,38 & $0,37>$ \\
\hline
\end{tabular}


MS no 04/102

\section{List of figures}

Figure 1. a) morphology context of LBBB waveforms; b) baseline wander; c) corrupted morphology context by d) muscle artefact; e) output of the filtering of the signal $c$; f) electrode motion artefact.

Figure 2. Principal Components Analysis on global contexts. The first axis $\left(\varphi^{1}=25.6 \%\right)$ presents a positive correlation (see Tab. 2) with the Ne values of ma noise, pvc, SNR $=15 \mathrm{~dB}$, etc which are difficult to process. In others words, the first axis reflects the positive contribution of the high $\mathrm{Ne}$ values (see section 3.3 for an explanation of the correlation coefficients). The second axis $\left(\varphi^{2}=11.6 \%\right)$ presents a positive correlation with $\mathrm{Ne}$ values of ma noise contexts and a negative correlation with Ne values of the em noise context (see Tab. 2). This axis opposes the ma and the em contexts.

Figure 3. Principal Components Analysis on morphology contexts corrupted with electrode motion artefact. The first axis $\left(\varphi^{1}=21.8 \%\right)$ presents a positive correlation with Ne values of signal contexts that have SNR $\geq-5 \mathrm{~dB}$ (see Tab. 3). The second axis $\left(\varphi^{2}=15.2 \%\right)$ presents a positive correlation with Ne values of noisy signal contexts $(\mathrm{SNR} \leq-5 \mathrm{~dB}$, see Tab. 3).

Figure 4. Principal Components Analysis on uncorrupted morphology contexts. The first axis $\left(\varphi^{1}=60.8 \%\right)$ is negatively correlated to the Ne values of the 4 detectors. The second axis $\left(\varphi^{2}=20.4 \%\right)$ is negatively correlated mostly to Ne values of AF2. 
MS no 04/102
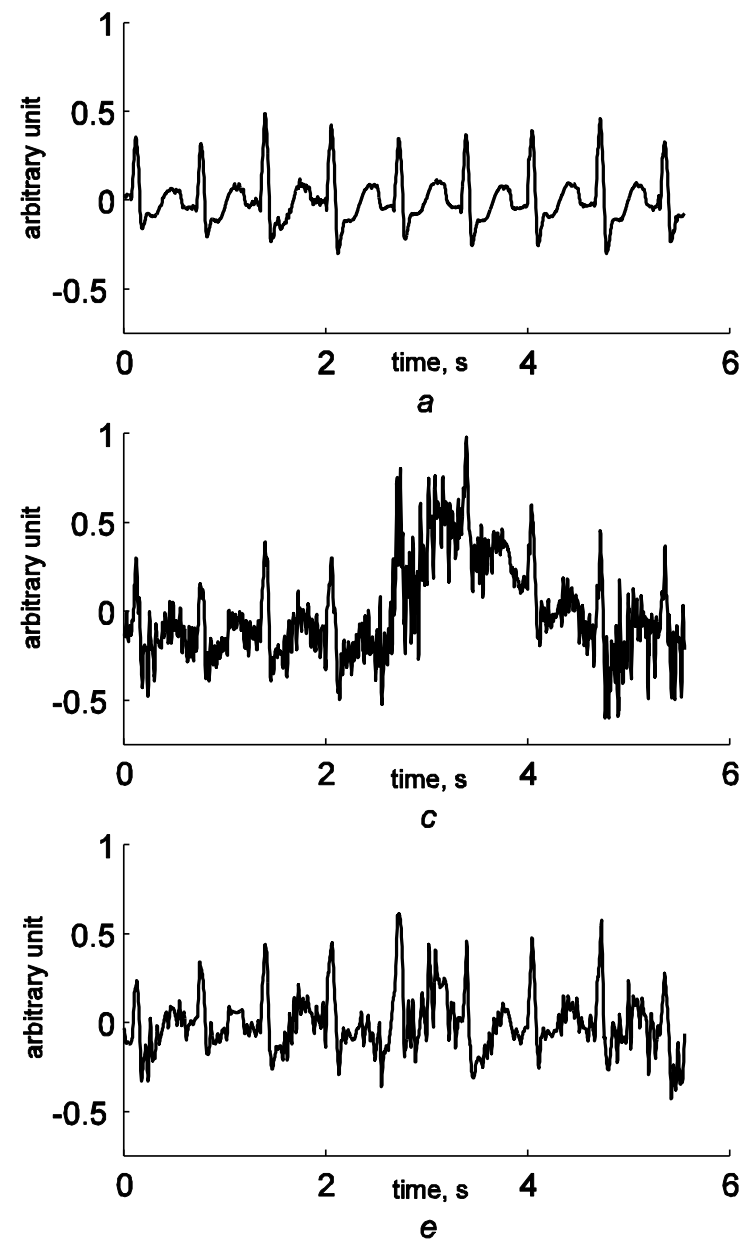
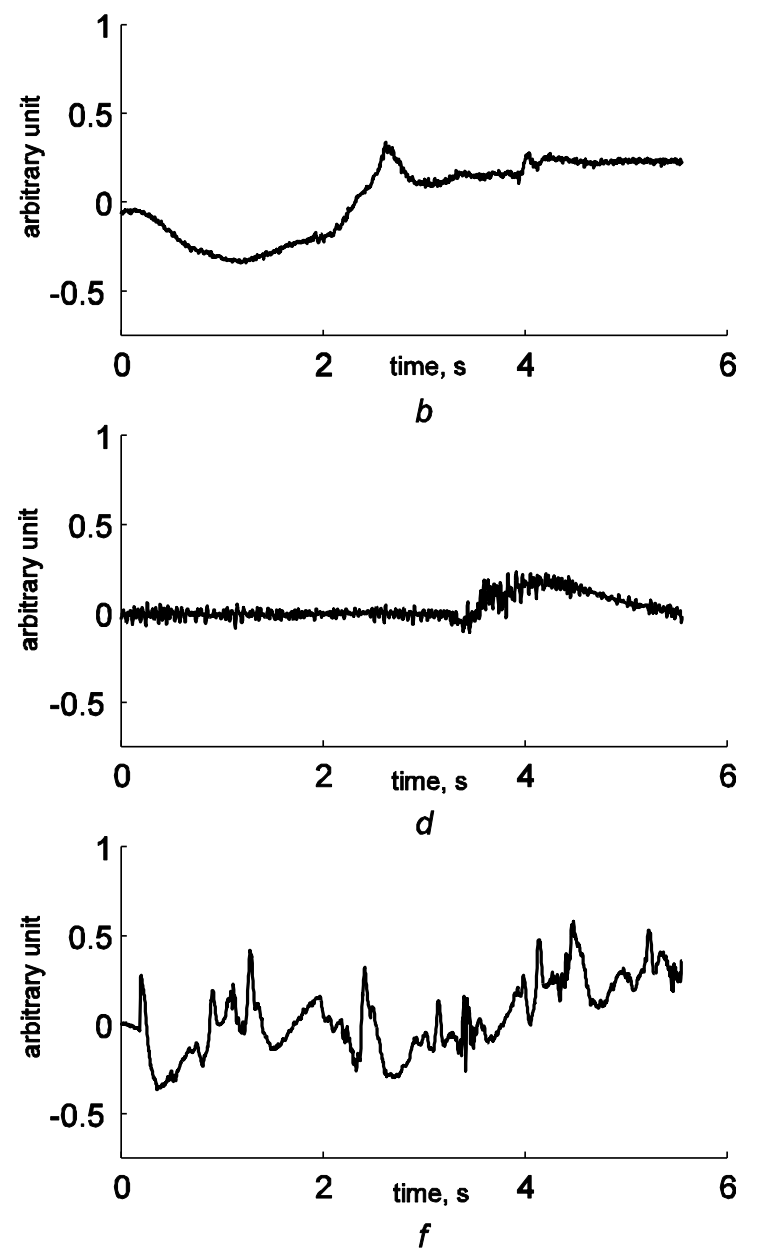

Figure 1

19 
MS no 04/102

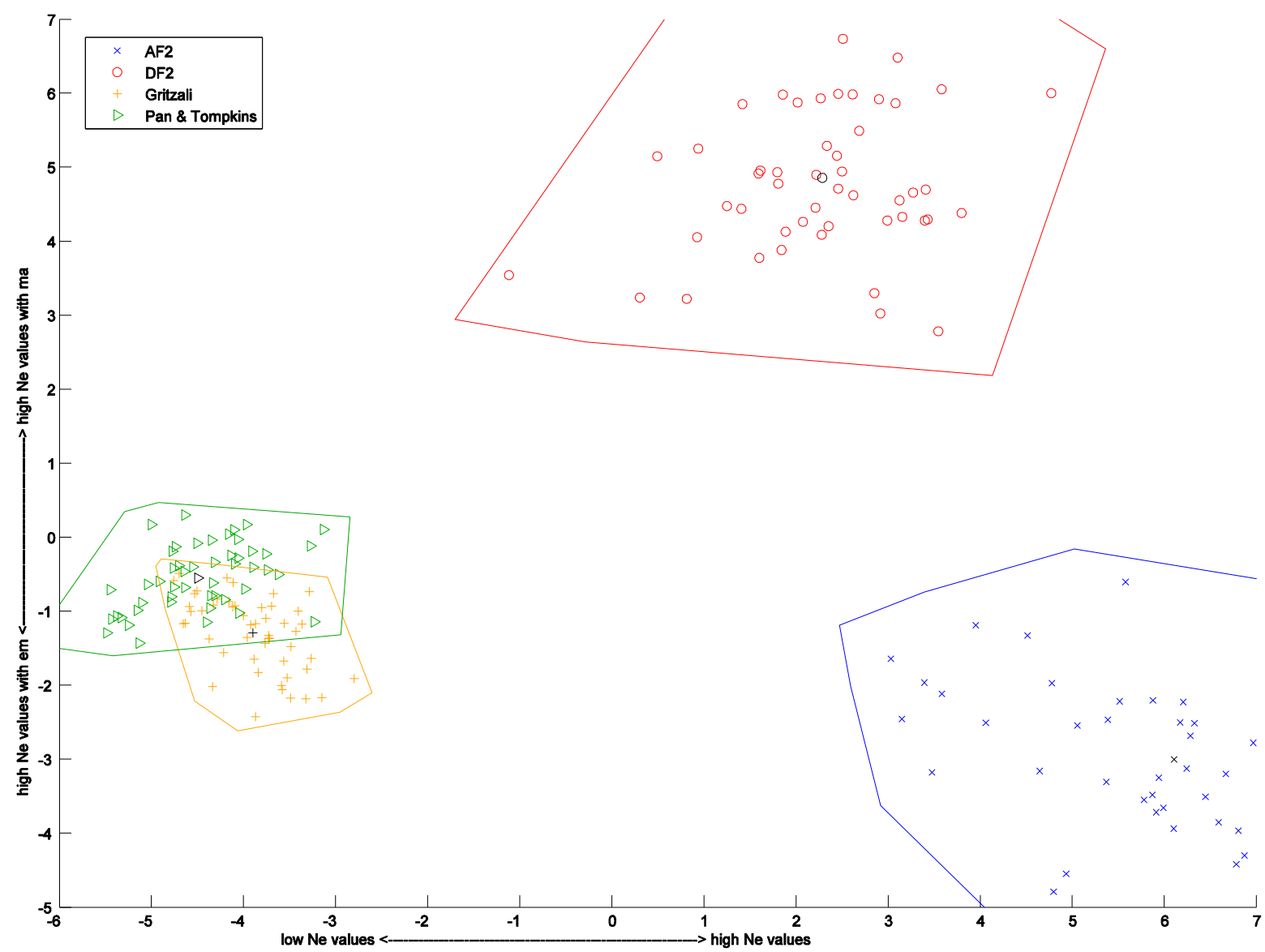

Figure 2 


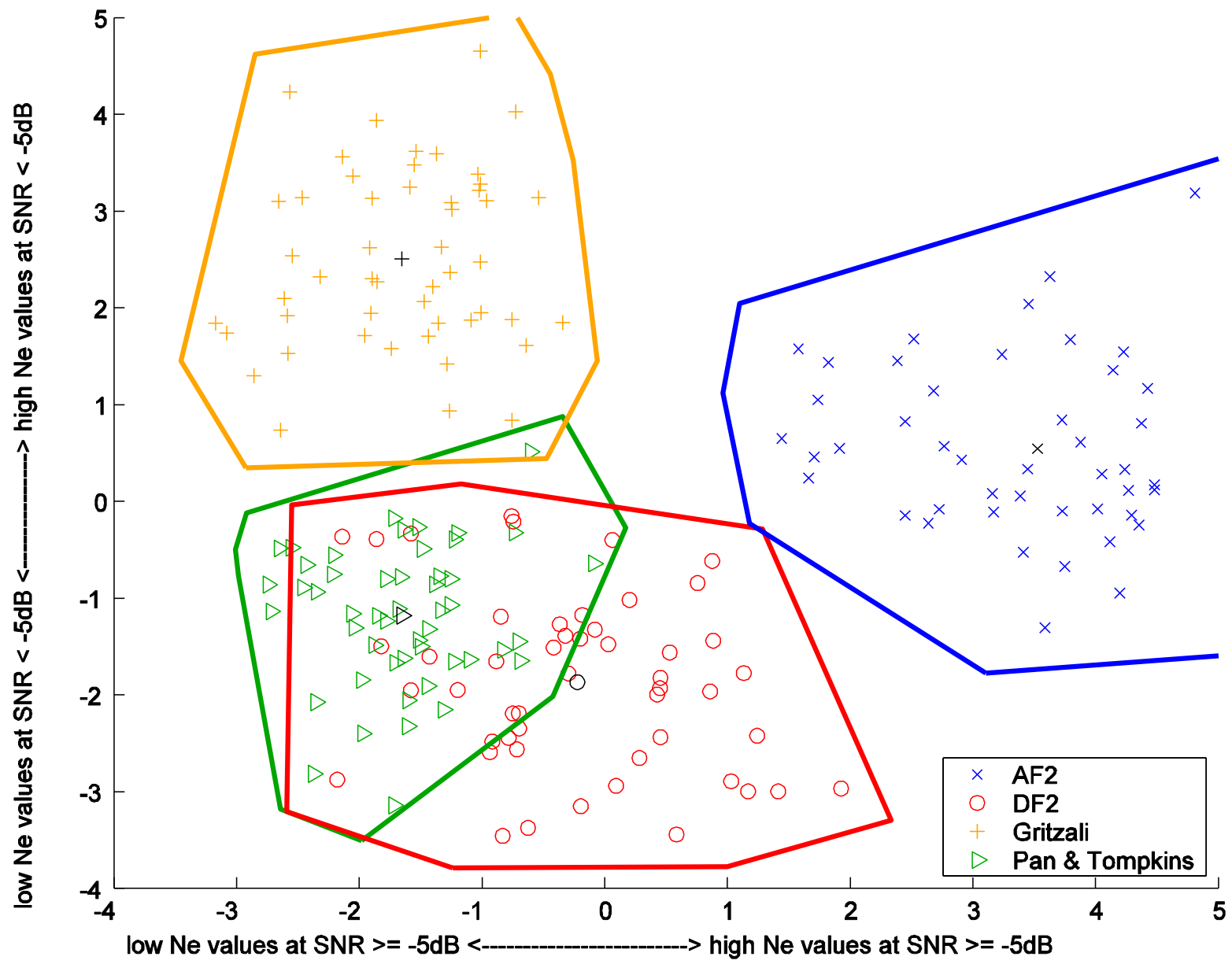

Figure 3 
MS no 04/102

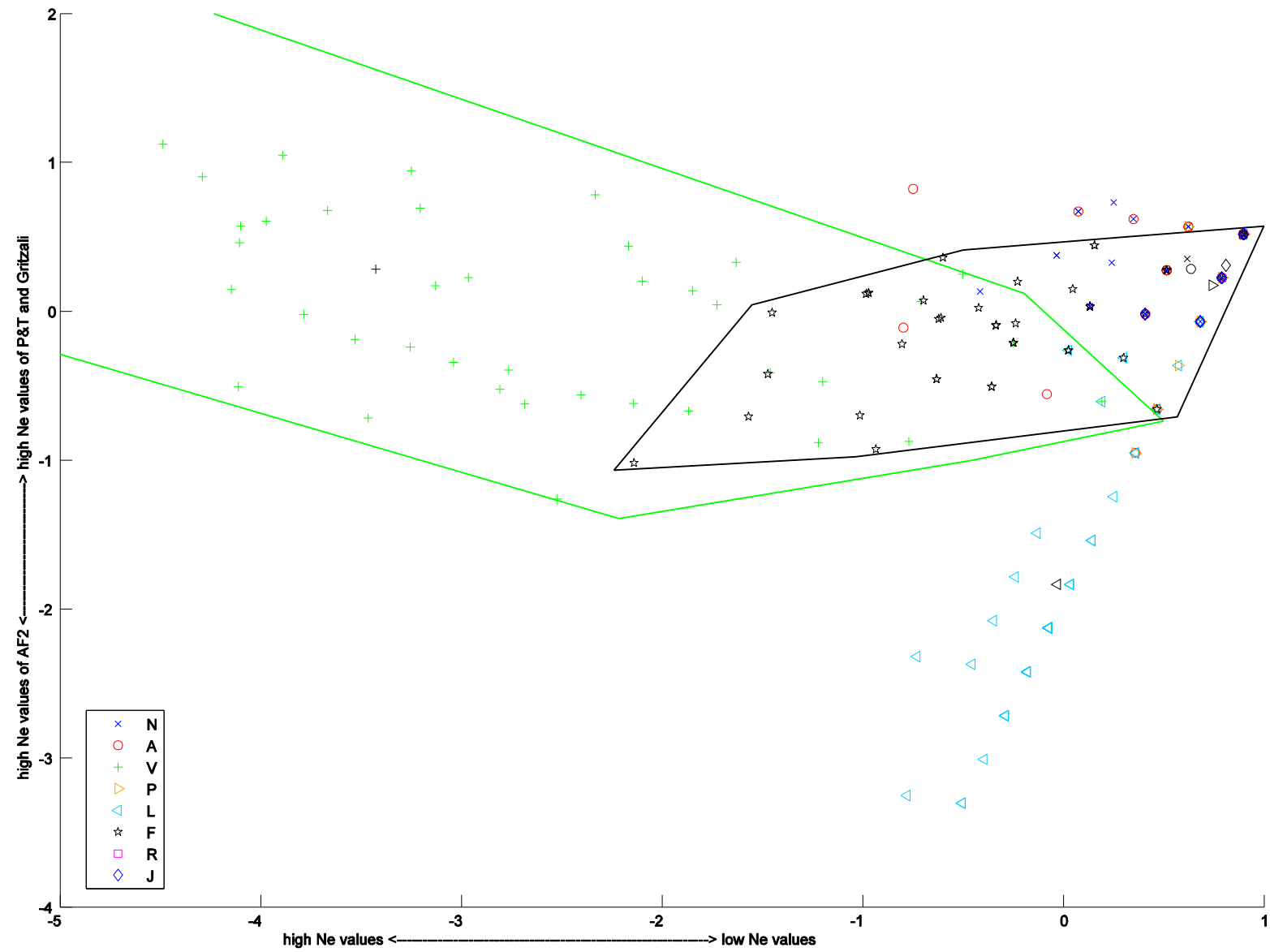

Figure 4 